Kragujevac Journal of Mathematics

Volume 39(2) (2015), Pages 127-132.

\title{
DEPENDENCE RESULT OF THE WEAK SOLUTION OF ROBIN BOUNDARY VALUE PROBLEMS
}

\author{
K. AKHLIL ${ }^{1}$
}

\begin{abstract}
In this article we establish an approximation result involving the Laplacian with Robin boundary conditions. It informs about the weak solution's dependence from the input function on the boundary.
\end{abstract}

\section{INTRODUCTION}

Let $\Omega$ be a bounded domain with Lipschitz boundary. We consider the problem of the Laplacian with Robin boundary conditions

$$
\frac{\partial u}{\partial \nu}+\beta u=0
$$

where $\nu$ is the outward normal vector and $\beta$ is a measurable positive bounded function on the boundary $\partial \Omega$. This kind of problems was extensively studied by many authors, we refer to $[1,2,3,5,8]$ and references therein for more details. Such boundary conditions appears in the modelisation of some physical, chimical or biological processes governed by the Laplacian equation.

Such processes are called Laplacian transport phenomena or diffusive transport phenomena. More in details, the diffusive transport phenomena describes the transport of species between two distinct "regions" separated by an interface. In biology, for example, it describes the process when water and minerals are pumped by roots from earths, or when ions and biological species penetrate through cellular membranes, or also when oxygen molecules diffuse towards and pass through alveolar ducts (see for example [6]).

The physical and biological properties of the interface described above are reproduced by the function $\beta$. What motivate this work is the fact that such functions

Key words and phrases. Robin boundary conditions, approximation.

2010 Mathematics Subject Classification. Primary: 65N99. Secondary: 58J90.

Received: 17 May, 2014.

Accepted: 4 August, 2015. 
can be too complicated to deal with. One can try then to approximate the function $\beta$ by a sequence of functions $\left(\beta_{n}\right)_{n \geq 0}$ more simple than $\beta$. The aim of this article is then to show a dependance result of a sequence of weak solutions $\left(u_{n}\right)_{n \geq 0}$ with a sequence of input functions $\left(\beta_{n}\right)_{n \geq 0}$. The proof is based on a technical Lemma due to Stampaccia [7].

\section{Preliminaries and Main Result}

We assume that $\Omega \subset \mathbb{R}^{d}(d \geq 3)$ is a bounded domain with Lipschitz boundary. We denote by $\sigma$ the restriction to $\partial \Omega$ of the $(d-1)$-dimensional Hausdorff measure.

We know that the following continuous embedding holds,

$$
H^{1}(\Omega) \rightarrow L^{q}(\Omega), \quad q=\frac{2 d}{d-2}
$$

Moreover each function $u \in H^{1}(\Omega)$ has a trace which is in $L^{s}(\partial \Omega)$, where $s=\frac{2(d-1)}{d-2}$; i.e., there is a constant $c>0$ such that

$$
\|u\|_{s, \partial \Omega} \leq c\|u\|_{H^{1}(\Omega)}, \quad \text { for all } u \in H^{1}(\Omega) .
$$

Let $\lambda>0$ be a real number, $f \in L^{p}(\Omega)(p>d)$ and $\beta$ be a nonnegative bounded measurable function on $\partial \Omega$. We consider the following Robin boundary value problem

$$
\begin{cases}-\Delta u+\lambda u=f, & \text { in } \Omega, \\ \frac{\partial u}{\partial \nu}+\beta u=0, & \text { in } \partial \Omega .\end{cases}
$$

The form associated with the Laplacian with Robin boundary condition is

$$
a_{\beta}(u, v)=\int_{\Omega} \nabla u \nabla v d x+\int_{\partial \Omega} \beta u v d \sigma, \quad \text { for all } u, v \in H^{1}(\Omega) .
$$

We start by the definition of the weak solution of the problem (2.3).

Definition 2.1. Let $f \in L^{p}(\Omega)$. For each $\lambda>0$, a function $u=G_{\beta}^{\lambda} f \in H^{1}(\Omega)$ is called a weak solution of the Robin boundary value Problem (associated with $\beta$ ) if for every $v \in H^{1}(\Omega)$

$$
a_{\beta}^{\lambda}(u, v)=\int_{\Omega} f v d x
$$

where for $u, v \in H^{1}(\Omega)$

$$
a_{\beta}^{\lambda}(u, v)=a_{\beta}(u, v)+\lambda \int_{\Omega} u v d x
$$

It is clear that the closed bilinear form $a_{\beta}$ is continuous on $H^{1}(\Omega)$ and also coercive on $H^{1}(\Omega)$ in the sense that there exists a constant $c>0$ such that for all $u \in H^{1}(\Omega)$

$$
a_{\beta}^{\lambda}(u, u) \geq\|u\|_{H^{1}(\Omega)}^{2} .
$$


Let $L$ be the linear functional on $H^{1}(\Omega)$ defined by: for $v \in H^{1}(\Omega)$

$$
L v:=\int_{\Omega} f v d x .
$$

Since $p \geq 2$, the functional $L$ is well defined and continuous on $H^{1}(\Omega)$. Thus by coerciveness of the bilinear form $a_{\beta}$, the Lax-Milgram Lemma (see [4, Corollaire V.8 p:84]) implies that there exists a unique weak solution $u \in H^{1}(\Omega)$ of the boundary value problem (2.3).

The following lemma is important in the proof of Theorem 2.2, we can find its proof in [7] Lemma 4.1.

Lemma 2.1. Let $\varphi=\varphi(t)$ be a nonnegative, nonincreasing function on the half line $t \geq k_{0} \geq 0$ such that there are positive constants $c, \alpha$ and $\delta(\delta>0)$ such that

$$
\varphi(h) \leq c(h-k)^{-\alpha} \varphi(k)^{\delta},
$$

for all $h>k \geq k_{0}$. Then we have

$$
\varphi\left(k_{0}+d\right)=0 \text {, where } d>0 \text { satisfies } d^{\alpha}=c \varphi\left(k_{0}\right)^{\delta-1} 2^{\delta(\delta-1)} .
$$

Theorem 2.1. Let $u$ be a weak solution and assume that $p>d$. Then

1) if $\lambda=0$ and $\Omega$ is of finite volume, there exists a strictly positive constants $C_{1}=C_{1}(d, p,|\Omega|)$ such that

$$
|u(x)| \leq C_{1}\|f\|_{p} \quad \text { a.e on } \bar{\Omega},
$$

2) if $\lambda>0$ and $\Omega$ is an arbitrary domain, there exist a strictly positive constant $C_{2}=C_{2}(d, p, \lambda)$ such that

$$
\left|G_{\beta}^{\lambda} f(x)\right| \leq C_{2}\|f\|_{p} \quad \text { a.e on } \bar{\Omega} .
$$

The proof can be found in [8] and is based on the Maza'ya inequality and a standard argument as in Theorem 4.1 of [7].

Our main result is the following Theorem.

Theorem 2.2. Any sequence $\left(u_{n}\right)_{n \geq 0}$ of weak solutions of the Robin boundary value problem associated to the sequence $\left(\beta_{n}\right)_{\geq 0}$ verify the following inequality:

$$
\left\|u_{n}-u_{m}\right\|_{\infty, \bar{\Omega}} \leq C\left\|u_{n}\right\|_{\infty, \partial \Omega}\left\|\beta_{n}-\beta_{m}\right\|_{\infty, \partial \Omega},
$$

for all $n, m \in \mathbb{N}$ and where $C$ may depend of $\lambda$.

\section{Proof of Theorem 2.2}

Proof. Let $\left(u_{n}\right)_{n \geq 0}$ be a sequence of weak solutions associated with the sequence $\left(\beta_{n}\right)_{\geq 0}$. Let $k \geq 0$ be a real number and define $u_{n, m}:=u_{n}-u_{m}$.

Define $v_{n, m}:=\left(\left|u_{n, m}\right|-k\right)^{+} \operatorname{sgn}\left(u_{n, m}\right)$. Then $v_{n, m} \in H^{1}(\Omega)$ and

$$
\nabla v_{n, m}= \begin{cases}\nabla u_{n, m}, & \text { in } A_{n, m}(k) \\ 0, & \text { otherwise }\end{cases}
$$


where $A_{n, m}(k)=\left\{x \in \bar{\Omega}:\left|u_{n, m}(x)\right|>k\right\}$. In the following, we write $u, v, A(k) \ldots$ instead of $u_{n, m}, v_{n, m}, A_{n, m}(k) \ldots$

It is clear that $a_{\beta_{n}}^{\lambda}\left(u_{n}, v\right)-a_{\beta_{m}}^{\lambda}\left(u_{m}, v\right)=0$. Calculating we obtain:

$$
\begin{aligned}
0= & \int_{\Omega} \nabla\left(u_{n}-u_{m}\right) \nabla v d x+\int_{\partial \Omega}\left(\beta_{n} u_{n}-\beta_{m} u_{m}\right) v d \sigma+\lambda \int_{\Omega}\left(u_{n}-u_{m}\right) v d x \\
= & \int_{A(k)}|\nabla v|^{2} d x+\int_{\partial \Omega}\left(\beta_{n}-\beta_{m}\right) u_{n}+\beta_{m}\left(u_{n}-u_{m}\right) v d \sigma+\lambda \int_{\Omega}\left(u_{n}-u_{m}\right) v d x \\
= & \int_{A(k)}|\nabla v|^{2} d x+\int_{\partial \Omega \cap A(k)}\left(\beta_{n}-\beta_{m}\right) u_{n} v d \sigma+\int_{\partial \Omega \cap A(k)} \beta_{m}\left(u_{n}-u_{m}\right) v d \sigma \\
& +\lambda \int_{A(k)}\left(u_{n}-u_{m}\right) v d x \\
= & \int_{A(k)}|\nabla v|^{2} d x+\int_{\partial \Omega \cap A(k)}\left(\beta_{n}-\beta_{m}\right) u_{n} v d \sigma+\int_{\partial \Omega \cap A(k)} \beta_{m} v^{2} d \sigma \\
& +k \int_{\partial \Omega \cap A(k)} \beta_{m}|v| d \sigma+\lambda \int_{A(k)} v^{2} d x+\lambda k \int_{A(k)}|v| d x \\
= & a_{\beta_{m}}^{\lambda}(v, v)+\int_{\partial \Omega \cap A(k)}\left(\beta_{n}-\beta_{m}\right) u_{n} v d \sigma+k \int_{\partial \Omega \cap A(k)} \beta_{m}|v| d \sigma+\lambda k \int_{A(k)}|v| d x
\end{aligned}
$$

It follows that

$$
\begin{aligned}
a_{\beta_{m}}^{\lambda}(v, v)+\int_{\partial \Omega \cap A(k)}\left(\beta_{n}-\beta_{m}\right) u_{n} v d \sigma & =-k \int_{\partial \Omega \cap A(k)} \beta_{m}|v| d \sigma-\lambda k \int_{A(k)}|v| d x \\
& \leq 0 .
\end{aligned}
$$

Which leads to

$$
a_{\beta_{m}}^{\lambda}(v, v) \leq \int_{\partial \Omega \cap A(k)}\left(\beta_{m}-\beta_{n}\right) u_{n} v d \sigma
$$

Using the Hölder inequality and (2.2), we obtain the following estimates,

$$
\begin{aligned}
a_{\beta_{m}}^{\lambda}(v, v) & \leq \int_{\partial \Omega \cap A(k)}\left(\beta_{m}-\beta_{n}\right) u_{n} v d \sigma \\
& \leq\left\|\beta_{n}-\beta_{m}\right\|_{\infty, \partial \Omega} \int_{\partial \Omega \cap A(k)} u_{n} v d \sigma \\
& \leq\left\|\beta_{n}-\beta_{m}\right\|_{\infty, \partial \Omega}\left\|u_{n}\right\|_{2, \partial \Omega \cap A(k)}\|v\|_{2, \partial \Omega \cap A(k)} \\
& \leq\left\|\beta_{n}-\beta_{m}\right\|_{\infty, \partial \Omega}\left\|u_{n}\right\|_{\infty, \partial \Omega}|\partial \Omega \cap A(k)|^{\frac{1}{2}}|\partial \Omega \cap A(k)|^{\frac{1}{2}-\frac{1}{s}}\|v\|_{s, \partial \Omega} \\
& \leq\left\|\beta_{n}-\beta_{m}\right\|_{\infty, \partial \Omega}\left\|u_{n}\right\|_{\infty, \partial \Omega}|\partial \Omega \cap A(k)|^{1-\frac{1}{s}}\|v\|_{s, \partial \Omega} \\
& \leq c\left\|\beta_{n}-\beta_{m}\right\|_{\infty, \partial \Omega}\left\|u_{n}\right\|_{\infty, \partial \Omega}|\partial \Omega \cap A(k)|^{1-\frac{1}{s}}\|v\|_{H^{1}(\Omega)} .
\end{aligned}
$$


We have then,

$$
\begin{aligned}
\alpha\|v\|_{H^{1}(\Omega)}^{2} & \leq a_{\beta_{m}}^{\lambda}(v, v) \\
& \leq c\left\|\beta_{n}-\beta_{m}\right\|_{\infty, \partial \Omega}\left\|u_{n}\right\|_{\infty, \partial \Omega}|\partial \Omega \cap A(k)|^{1-\frac{1}{s}}\|v\|_{H^{1}(\Omega)} .
\end{aligned}
$$

It follows that

$$
\|v\|_{H^{1}(\Omega)} \leq c_{1}\left\|\beta_{n}-\beta_{m}\right\|_{\infty, \partial \Omega}\left\|u_{n}\right\|_{\infty, \partial \Omega}|\partial \Omega \cap A(k)|^{1-\frac{1}{s}} .
$$

Using the inequalities (2.1) and (2.2), we obtain the following estimates,

$$
\|v\|_{s, \partial \Omega \cap A(k)} \leq c_{2}\left\|\beta_{n}-\beta_{m}\right\|_{\infty, \partial \Omega}\left\|u_{n}\right\|_{\infty, \partial \Omega}|\partial \Omega \cap A(k)|^{1-\frac{1}{s}}
$$

and

$$
\|v\|_{q, A(k)} \leq c_{3}\left\|\beta_{n}-\beta_{m}\right\|_{\infty, \partial \Omega}\left\|u_{n}\right\|_{\infty, \partial \Omega}|\partial \Omega \cap A(k)|^{1-\frac{1}{s}} .
$$

Let now $h>k \geq 0$. Then $A(h) \subset A(k)$ and on $A(h)$ we have $|v| \geq h-k$. It follows that

$$
\begin{aligned}
\|v\|_{s, \partial \Omega \cap A(k)} & \geq\|v\|_{s, \partial \Omega \cap A(h)} \\
& \geq\||u|-k\|_{s, \partial \Omega \cap A(h)} \\
& \geq(h-k)|\partial \Omega \cap A(h)|^{\frac{1}{s}} .
\end{aligned}
$$

We deduce from (3.1) that

$$
(h-k)|\partial \Omega \cap A(h)|^{\frac{1}{s}} \leq c_{2}\left\|\beta_{n}-\beta_{m}\right\|_{\infty, \partial \Omega}\left\|u_{n}\right\|_{\infty, \partial \Omega}|\partial \Omega \cap A(k)|^{1-\frac{1}{s}}
$$

which reduces to,

$$
|\partial \Omega \cap A(h)| \leq c_{2}^{s}(h-k)^{-s}\left\|\beta_{n}-\beta_{m}\right\|_{\infty, \partial \Omega}^{s}\left\|u_{n}\right\|_{\infty, \partial \Omega}^{s}|\partial \Omega \cap A(k)|^{s-1} .
$$

Set $\phi(h)=|\partial \Omega \cap A(h)|$, we obtain,

$$
\phi(h) \leq C(h-k)^{-s} \phi(k)^{s-1},
$$

where $C=c_{2}^{s}\left\|\beta_{n}-\beta_{m}\right\|_{\infty, \partial \Omega}^{s}\left\|u_{n}\right\|_{\infty, \partial \Omega}^{s}$.

As $s-1>1$, then the conditions of the Lemma 2.1 are satisfied with $\delta=s-1$ and $k_{0}=0$, one obtain $\phi(d)=0$ where $d>0$ satisfies $d^{s}=C \phi(0)^{s-2} 2^{(s-1)(s-2)}$, consequently

$$
d=c_{4}\left\|\beta_{n}-\beta_{m}\right\|_{\infty, \partial \Omega}\left\|u_{n}\right\|_{\infty, \partial \Omega}
$$

and

$$
\left\|u_{n}-u_{m}\right\|_{\infty, \partial \Omega} \leq c_{4}\left\|u_{n}\right\|_{\infty, \partial \Omega}\left\|\beta_{n}-\beta_{m}\right\|_{\infty, \partial \Omega} .
$$

In the same way as in (3.3), we obtain

$$
\|v\|_{q, A(k)} \geq(h-k)|A(k)|^{\frac{1}{q}} .
$$

From (3.2), we deduce

$$
(h-k)|A(h)|^{\frac{1}{q}} \leq c_{3}\left\|\beta_{n}-\beta_{m}\right\|_{\infty, \partial \Omega}\left\|u_{n}\right\|_{\infty, \partial \Omega}|\partial \Omega \cap A(k)|^{1-\frac{1}{s}} .
$$


We take $k=d$ and $h=\gamma d$ with $\gamma>1$, we obtain $|A(\gamma d)|=0$ which leads to

$$
\begin{aligned}
\left\|u_{n}-u_{m}\right\|_{\infty, \Omega} & \leq \gamma d \\
& \leq \gamma c_{4}\left\|u_{n}\right\|_{\infty, \partial \Omega}\left\|\beta_{n}-\beta_{m}\right\|_{\infty, \partial \Omega} .
\end{aligned}
$$

From (3.4) and (3.5) we obtain our Theorem.

Corollary 3.1. Let $\left(u_{n}\right)_{n \geq 0}$ be a sequence weak solutions associated with the sequence $\left(\beta_{n}\right)_{\geq 0} \in L^{\infty}(\partial \Omega)$ such that $\inf _{n} \beta_{n}>0$ then if $\left(u_{n}\right)_{n \geq 0}$ is uniformly bounded we have for $p>d$

$$
\left\|u_{n}-u_{m}\right\|_{\infty, \bar{\Omega}} \leq C\|f\|_{p}\left\|\beta_{n}-\beta_{m}\right\|_{\infty, \partial \Omega},
$$

for all $n, m \in \mathbb{N}$ and where $C$ may depend of $\lambda$.

In the case where the sequence of weak solutions $\left(u_{n}\right)_{n \geq 0}$ is uniformly bounded with respect to $n$ we have the following consequence

Corollary 3.2. Let $\left(u_{n}\right)_{n \geq 0}$ be a sequence weak solutions associated with the sequence $\left(\beta_{n}\right)_{\geq 0} \in L^{\infty}(\partial \Omega)$ such that $\inf _{n} \beta_{n}>0$ and $\lim _{n} \beta_{n}(x)=\beta(x)$ a.e $x \in \partial \Omega$ then if $\left(u_{n}\right)_{n \geq 0}$ is uniformly bounded we have $\lim _{n} u_{n}(x)=u(x)$ a.e $x \in \bar{\Omega}$, where $u$ is the weak solution associated with $\beta$.

Acknowledgments. I would like to thank Markus Kunze and Sultana Ben Aadi for proposing the question answered in this article and the anonymous referee for valuable remarks.

\section{REFERENCES}

[1] K. Akhlil, Probabilistic solution of the general Robin boundary value problem on arbitrary domains, Int. J. Stoch. Anal. (2012), Article ID 163096, 17 pages.

[2] K. Akhlil, The Laplacian with general Robin boundary conditions involving signed measures, http://arxiv.org/pdf/1303.5572v1.pdf, 2013, Submitted to Acta Math. Appl. Sin.

[3] W. Arendt and M. Warma, The Laplacian with Robin boundary conditions on arbitrary domains, Potential Anal. 19 (2003), 341-363.

[4] H. Brezis, Analyse Fonctionnelle. Théorie et Application, Masson, Paris, 1983.

[5] D. Daners, Robin boundary value problems on arbitrary domains, Trans. Amer. Math. Soc. 352 (2000), 4207-4236.

[6] D. S. Grebenkov, Partially reflected Brownian motion: a stochastic approach to transport phenomena. in Velle, L. R. ed. Focus on Probability Theory, Nova Science Publishers, 2006, 135-169.

[7] G. Stampacchia, Equation elliptiques du second ordre à coefficients discontinus, Séminar sur les equations aux dérivée partielles, Collège de France, 1963.

[8] M. Warma, The Laplacian with General Robin Boundary Conditions, Ph.D. Dissertation, University of Ulm, 2002.

\footnotetext{
${ }^{1}$ Insitut of Applied Analysis,

UNIVERSITY OF ULM,

89069 Ulm, GERMANY

E-mail address: akhlil.khalid@gmail.com
} 